\title{
BANDWIDTH REDUCTION ON SPARSE MATRICES BY INTRODUCING NEW VARIABLES
}

\section{REDUCCIÓN DEL ANCHO DE BANDA DE MATRICES DISPERSAS MEDIANTE LA INTRODUCCIÓN DE NUEVAS VARIABLES}

\author{
Rainer Glüge ${ }^{1}$ \\ Recibido 2 de marzo de 2010, aceptado 19 de noviembre de 2010 \\ Received: March 2, 2010 Accepted: November 19, 2010
}

\begin{abstract}
RESUMEN
Se analiza un método para la reducción del ancho de banda de matrices dispersas, el cual consiste en fraccionar ecuaciones, substituir e introducir nuevas variables, similar a la descomposición en subestructuras utilizada en el método de los elementos finitos (FEM). Es especialmente útil si el ancho de banda no puede ser reducido intercambiando estratégicamente columnas y líneas. En estos casos, dividir ecuaciones y reordenar líneas y columnas puede reducir el ancho de banda, al costo de introducir nuevas variables. En comparación con el método de las subestructuras en el FEM, en el cual la descomposición está hecha antes de obtener la matriz del sistema, la metodología que se presenta está aplicada después de obtener el sistema lineal, independiente de su origen. El método está aplicado con éxito en una matriz dispersa en el contexto del FEM, lo cual resulta en un aumento de eficiencia del algoritmo directo para resolver el sistema lineal.
\end{abstract}

Palabras clave: Matriz dispersa, ancho de banda, elemento de volumen representativo, homogenización, condiciones de borde cinemáticamente mínimas.

\section{ABSTRACT}

A sparse matrix bandwidth reduction method is analyzed. It consists of equation splitting, substitution and introducing new variables, similar to the substructure decomposition in the finite element method (FEM). It is especially useful when the bandwidth cannot be reduced by strategically interchanging columns and rows. In such cases, equation splitting and successive reordering can further reduce the bandwidth, at cost of introducing new variables. While the substructure decomposition is carried out before the system matrix is built, the given approach is applied afterwards, independently on the origin of the linear system. It is successfully applied to a sparse matrix, the bandwidth of which cannot be reduced by reordering. For the exemplary FEM simulation, an increase of performance of the direct solver is obtaine.

Keywords: Sparse matrix, bandwidth, representative volume element (RVE), homogenization, kinematic minimal boundary conditions.

\section{INTRODUCTION}

A linear system, conveniently denoted in a matrix-vector notation

$$
\left[\begin{array}{lll}
A_{11} & \cdots & A_{1 n} \\
\vdots & & \vdots \\
A_{n 1} & \cdots & A_{n n}
\end{array}\right]\left[\begin{array}{c}
x_{1} \\
\vdots \\
x_{n}
\end{array}\right]=\left[\begin{array}{c}
y_{1} \\
\vdots \\
y_{n}
\end{array}\right]
$$

is invariant under the simultaneous interchange of $x_{i}$ and $x_{j}$ and the columns $i$ and $j$ of the matrix, and the simultaneous interchange of $y_{i}$ and $i_{j}$ and the rows $i$ and $j$ of the matrix. Depending on the solution algorithm that should be applied, different matrix characteristics may be advantageous. If, for example, Gaussian elimination is used, the creation of non-zero entries (fill in) during the process can be reduced either by reordering the system such that the non-zero entries concentrate on the main diagonal and columns that range upwards from it, or by concentrating the non-zero entries in a band around the main diagonal. The latter case corresponds to a reduction of the bandwidth of the matrix. The bandwidth of a matrix is related to the maximum distance of non-zero matrix entries from the main diagonal. One distinguishes the left and the right half bandwidth,

\footnotetext{
1 Institut für Mechanik, Otto-von-Guericke-Universität Magdeburg. Universitätsplatz 2, D-39106, Magdeburg, Germany. E-mail:gluege@ ovgu.de
} 


$$
\begin{aligned}
& k_{1}=\max (i-j), \quad i>j, \quad A_{i j} \neq 0 \\
& k_{2}=\max (j-i), \quad j>i, \quad A_{i j} \neq 0
\end{aligned}
$$

which coincide for symmetric matrices. The bandwidth $b$ is given by $b=k_{1}+k_{2}$. Especially direct solution algorithms can take advantage of a band structure, which is moreover helpful in reducing the memory requirements. For direct solvers, $t_{\mathrm{cpu}} \approx c b^{2}$ holds. Consequently, one is interested in reordering the linear system such that the system matrix bandwidth is reduced.

The efficient reordering of linear systems is an important topic discussed in the literature since sparse linear systems emerged routinely in engineering applications, i.e. since the development of the finite difference and the finite element method and the availability of computers. The reordering such that $b$ is minimized is a combinatorial problem. Different algorithms that base on a graph representation of the non-zero connections of columns and rows have been proposed. The most common methods are the CuthillMcKee and the Reverse Cuthill-McKee algorithm [6-5], Sloans ordering [14], Gibbs-Poole-Stockmeyer ordering [10], minimum degree ordering and nested dissection, which is also referred to as nested substructure method in the context of the FEM [7]. A survey is given by [2]. However, there are cases in which the bandwidth cannot be reduced by reordering. If the linear system consists mostly of equations with a small number of terms but at least one equation has a considerably larger number of terms, the matrix contains dominant non-zero rows, while the matrix contains dominant non-zero columns if at least one variable appears much more often in the equations than the other variables. Such linear systems are not encountered very often, but they can arise, e.g., if a large number of nodes of a finite element mesh is constrained by few equations. Here, an approach which permits a further reduction of the bandwidth at the cost of the overall system size is presented, and tested in conjunction with the FE system ABAQUS.

\section{BANDWIDTH REDUCTION BY INTRODUCING NEW DEGREES OF FREEDOM}

\author{
Row-Dominant matrices \\ Consider the linear system
}

$$
\left[\begin{array}{cccc}
A_{11} & A_{12} & \cdots & A_{1 n} \\
& A_{22} & & \\
& & \ddots & \\
& & & A_{n n}
\end{array}\right]\left[\begin{array}{c}
x_{1} \\
\vdots \\
x_{n}
\end{array}\right]=\left[\begin{array}{c}
y_{1} \\
\vdots \\
y_{n}
\end{array}\right]
$$

i.e. a matrix with the first row and the diagonal entirely filled with non-zero entries, while all other matrix elements are equal to zero. The bandwidth of the matrix is equal to $n$, and one can see that the interchanging of columns and rows can reduce the bandwidth maximally to approximately $n / 2$, by putting the dominant row in a more central position. Let us introduce the substitution

$$
x^{*}=A_{1 m+1} x_{m+1}+\ldots+A_{1 n} x_{n}
$$

and treat $x^{*}$ as unknown. Hence, we add the latter equation to the list of equations and rewrite the system as

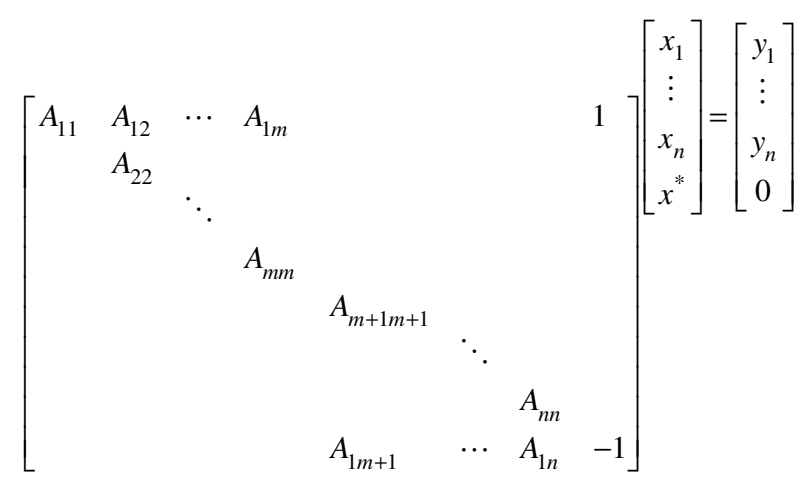

The matrix bandwidth can now be reduced by interchanging row and columns to approximately $n / 4$. The new system has one more degree of freedom, but its minimal bandwidth is halved. The procedure can be applied to the remaining dominant rows to reduce the bandwidth to a certain value, or one can directly split the first equation into $p$ equations, reducing the bandwidth to approximately $n /(2 p)$. 


\section{Column-Dominant matrices}

A similar strategy can be employed for column-dominant linear systems. Consider the linear system

$$
\left[\begin{array}{llll}
A_{11} & & & \\
A_{21} & A_{22} & & \\
\vdots & & \ddots & \\
A_{n 1} & & & A_{n n}
\end{array}\right]\left[\begin{array}{c}
x_{1} \\
\vdots \\
x_{n}
\end{array}\right]=\left[\begin{array}{c}
y_{1} \\
\vdots \\
y_{n}
\end{array}\right]
$$

The first column can be split up by introducing $x^{*}{ }_{1}=x_{1}$, and distributing the coefficients that are connected to $x_{1}$ equally on $x_{1}$ and $x_{1}{ }^{*}$. Adding the equation and the new variable to the system, one obtains

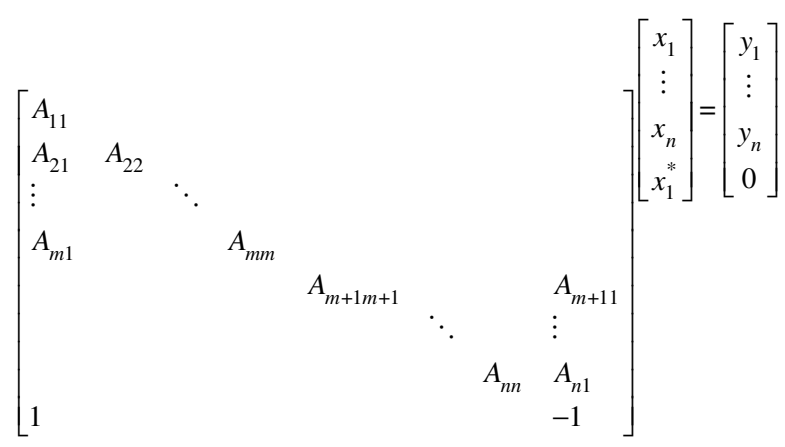

Again, the bandwidth of the latter system can be reduced by column and row permutation.

\section{PRESERVATION OF SYMMETRY}

The latter substitutions can be carried out such that the symmetry is preserved, which is demonstrated on a symbolically filled matrix. The vector reordering is disregarded for convenience. Being given a matrix of the form

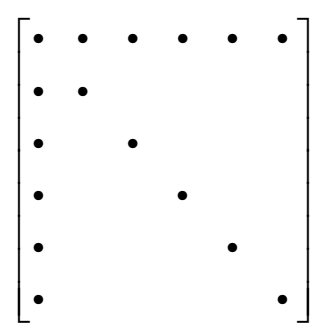

we firstly split the dominant column and append the newly formed column and row,

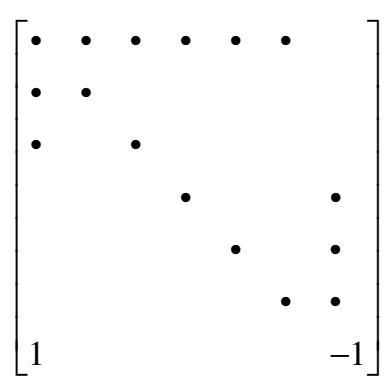

and then split the dominant row and append the newly formed column and row,

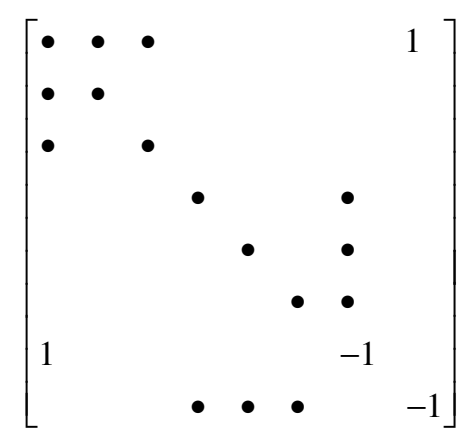

followed by interchanging the newly added columns or rows (rows here)

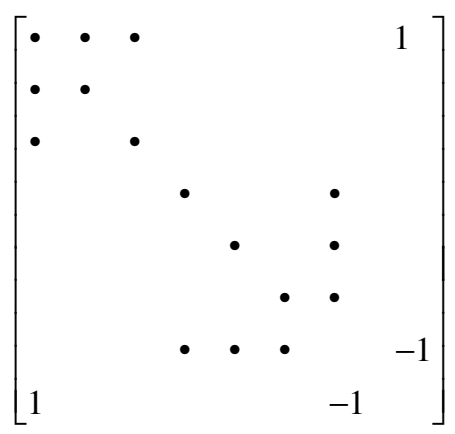

For large systems, the increase in variables may have no practical effect at all, converse to the bandwidth reduction. For the preceding examples, the equation splitting is more costly than applying Gaussian elimination, after reordering the matrices such that the fill in is avoided. However, in some cases, the splitting of dominant rows and columns can significantly reduce the solution effort. In the following section an example for the profitable application of the equation splitting is given. 


\section{EXAMPLE}

The finite element method is used to approximate the solution of a partial differential (PDE) equation by discretizing the domain by finite elements, which are connected at nodes. The solution is approximated by piecewise steady functions inside the elements, the parameters of which are determined by exploiting the weak form of the PDE (see, e.g., [1]). The smallest possible bandwidth of the symmetric system matrix depends on the number of elements to which the node with the most connections is connected. The actual bandwidth depends on the specific structure of the finite element mesh. Reordering the nodes corresponds to column and row interchanging. There are geometries for which even an optimized mesh structure has a large bandwidth. But even in such cases the bandwidth is usually considerably smaller than the system size. However, the FEM permits a connection of nodes not only by the elements, but by other constraint equations.

Note that in the context of the FEM, the algorithm demonstrated here is similar to the decomposition of the FE model into hyper- and substructures. Referring to the substitution (3), one would say that the nodes belonging to the $x_{i}$ that are summarized to $x^{*}$ form a substructure. The procedure discussed here does not operate on nodes but on degrees of freedom. The most important difference is that the method presented here is independent on the problem, i.e. it can be applied algorithmically to any linear system, while the substructure decomposition is part of the specific FE modelling. In the substructure decomposition, the structure is divided into independent substructures, while the substitution (3) must not be a reasonable division into independent parts from the engineering point of view. We encountered the problem when we prescribed the average displacement $\bar{u}$ on an entire face of a structure in a continuum mechanics problem, which results in a large constraint equation

$$
u_{1}+u_{2}+\ldots+u_{n}=n \bar{u}
$$

In our case, the constraints emerge in a homogenization procedure. Homogenization bridges the gap from one scale to a larger scale. If one knows the constituents of a microstructure and their material properties, one can approximate the behaviour on a larger scale by averaging over the volume on the lower scale (see, e.g., [4] and the references within). Here, we present a numerical example. We want to apply an average deformation gradient

$$
\bar{F}=\frac{1}{V} \int_{\Omega} F(X) d V
$$

to a cubic representative volume element (RVE). $\Omega$ denotes the domain occupied by the body in the reference placement. $\boldsymbol{F}$ and $\boldsymbol{H}$ are called the deformation and the displacement gradient, respectively, $\boldsymbol{x}$ and $\boldsymbol{X}$ are the position vectors of material points in the reference and the current placement, and $\boldsymbol{u}=\boldsymbol{x}-\boldsymbol{X}$ denotes the displacement vector. For an account to continuum mechanics see for example [12,3].

$$
F=x(X) \otimes \nabla_{X}=(X+u(X)) \otimes \nabla_{X}=I+H
$$

$\bar{F}$ is commonly enforced by homogeneous or periodic boundary conditions. These have the drawback that they stiffen the RVE artificially, as, e.g., shear bands cannot arrange freely. Here we focus on applying $\bar{F}$ without further constraints, which is referred to as the kinematic minimal boundary conditions [13], natural boundary conditions [8] or weakly enforced kinematic boundary conditions [9]. By Gauss theorem we convert the volume integral into a surface integral,

$$
\begin{gathered}
\bar{F}=\frac{1}{V} \int_{\Omega}(I+u(X)) \otimes \nabla_{X} d V \\
I+\bar{H}=\frac{1}{V} V I+\frac{1}{V} \int_{\Omega} u(X) \otimes n_{0} d A \\
\bar{H}=\frac{1}{V} \int_{\Omega} u(X) \otimes n_{0} d A
\end{gathered}
$$

In the FE implementation, the latter integral converts into a sum over the weighted displacements of the surface nodes, the weight of which depends on the fraction of the surface that is assigned to each node. E.g., for hexahedral elements, which result in quadrilateral surfaces, a fourth of the surface of each element to which the node is connected is added. The FE system ABAQUS has been used for the following example. The FE model consists of a regular meshed cube (20 elements per edge), linear eight node bricks (element type C3D8) are used. The corner node at $(0,0,0)$ is tied, which is the only direct displacement boundary condition. The deformation is enforced by prescribing the $\bar{H}$ as described above. For this purpose, 3 artificial nodes have been created, the 3 degrees of 
freedom of which represent the components of $\bar{H}$. In any case, 9 large constrained equations have to be taken into account. It remains open whether the artificial nodes are constrained by a displacement (average straining) or by a force (average stress). For the comparison between not splitting and splitting of the equations and for checking of the implementation, a homogeneous linear elastic isotropic material behaviour is assumed (St. Venant-Kirchhoff). For illustration purposes of the boundary conditions, a central hard spherical inclusion of diameter $0.4 *$ edge length and $\mathrm{G}_{\text {sphere }}=5 \mathrm{G}_{\text {matrix }}=\mathrm{G}_{\text {sphere }}=5 \mathrm{G}_{\text {matrix }}=10.000 \mathrm{MPa}$ (shear and compression modulus, respectively) is included by the Gauss-point method (multiphase elements, see [11]). With this RVE, a uniaxial tension and a shear test have been carried out. For the uniaxial tension, $\bar{H}_{11}=0.1$ and $\bar{H}_{12}=\bar{H}_{13}=\bar{H}_{23}=\bar{H}_{21}=\bar{H}_{31}=\bar{H}_{32}=0$ have been prescribed, i.e. seven large constraining equations are included. The components $\bar{H}_{22}$ and $\bar{H}_{33}$ are not constrained in order to permit an average lateral straining. For the shear test, $\bar{H}_{22}$ and $\bar{H}_{13}=\bar{H}_{23}=\bar{H}_{21}=\bar{H}_{31}=\bar{H}_{32}=0$ haven been prescribed, while the average normal straining can freely adjust, i.e. $\bar{H}_{11}, \bar{H}_{22}$ and $\bar{H}_{33}$ are not prescribed.

Table 1 gives an overview on the difference between the FE simulations if carried out with and without equation splitting. Both simulation give exactly the same results and convergence behaviour, since the modifications of the linear system presented here do not affect the results. However, one can see in Table 1 that the equation splitting results in a considerable reduction of linear system solver effort. In Figure 1, the deformed RVE with the spherical inclusion is depicted. For a review of the FE simulations, supplementary files are provided at http://www.ovgu. de/ifme/l-festigkeit/ Rev_chilena_de_eng_supplement_ gluege_2010.zip, including the ABAQUS input files, the user material subroutine, the iteration log files and the output databases.

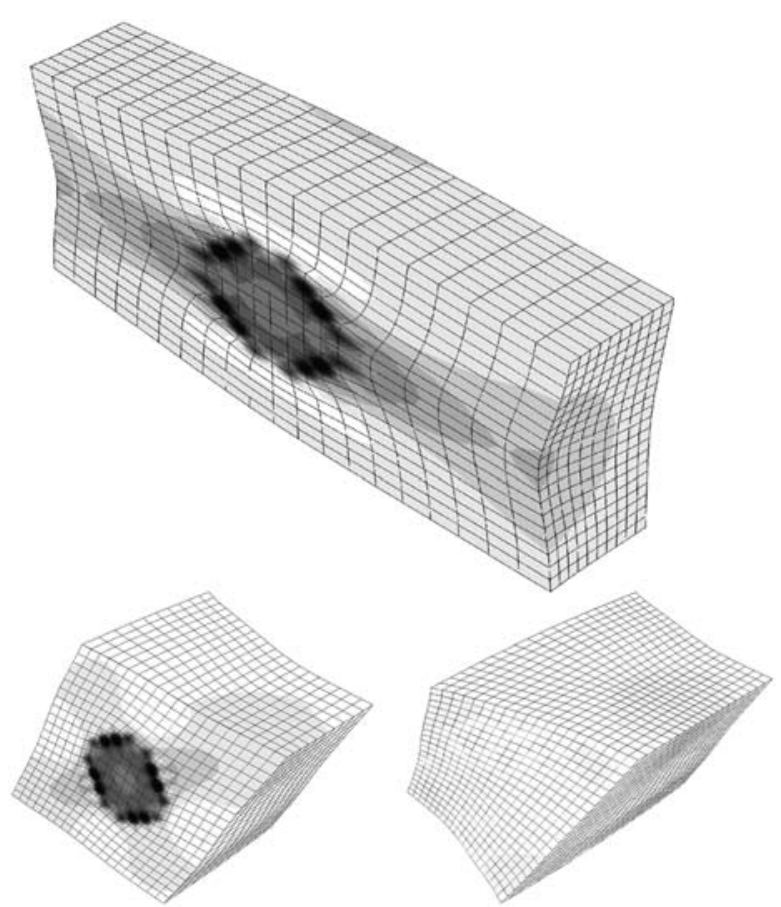

Figure 1. Cross sections of two deformed RVE with a central spherical inclusion. For the tension test (top), the displacement is scaled uniformly by a factor of 10 in order to amplify the deformation. The greyscaling (12 bands) corresponds to the equivalent Mises stress, from $400 \mathrm{MPa}$ (white) to $1.200 \mathrm{MPa}$ (black). For the shear test (bottom), the displacement is scaled by a factor of 2 in the shear direction (d) and the shear plane normal (n), and by a factor of 20 in the direction normal to $\mathrm{d}$ and $\mathrm{n}$. The greyscaling (12 bands) corresponds to the equivalent Mises stress, from 1.000MPa (white) to $2.400 \mathrm{MPa}$ (black). One can see the non-periodicity of the deformation.

Table 1. Long constraining equations vs. equation splitting with a direct solver in ABAQUS.

\begin{tabular}{|l|c|c|}
\hline & Splitting & No splitting \\
\hline Max. Terms in eq & 20 & $2 * 21^{2}+1=883$ \\
\hline Deg. Of freedom & 28215 & $3 * 21^{3}+9=27.792$ \\
\hline $\begin{array}{l}\text { Floating point ops per } \\
\text { iteration }\end{array}$ & $4.61 \mathrm{E} 10$ & $4.34 \mathrm{E} 11$ \\
\hline Min. Memory usage & $256 \mathrm{MB}$ & $853.71 \mathrm{MB}$ \\
\hline $\begin{array}{l}\text { FE sim wallclock } \\
\text { time }\end{array}$ & 141 & 1.621 \\
\hline
\end{tabular}




\section{CONCLUSIONS}

The present work points out problems that may emerge when row- and column-dominant linear systems are treated by direct solution methods. An efficient treatment is exemplified on a continuum mechanics problem, namely a numerical homogenization by the representative volume element technique, where kinematic minimal boundary conditions have been employed. Further research may focus on how the modifications affect the properties of the linear system. Moreover, it should not be concealed that the kinematic minimal boundary conditions are not as commonly employed as the periodic displacement and the homogeneous displacement boundary conditions, and have received therefore less attention. In particular, the question under which circumstances the kinematic minimal boundary conditions satisfy the Hill condition [15] is not answered conclusively.

\section{ACKNOWLEDGEMENT}

I like to express my gratitude to my wife, who reviewed the Spanish part of this work.

\section{REFERENCES}

[1] T. Belytschko, W.K. Liu and B. Moran. "Nonlinear Finite Elements for Continua and Structures". John Wiley \& Sons. New York, USA. 2000.

[2] M. Benzi. "Preconditioning Techniques for Large Linear Systems: A Survey". Journal of Computational Physics. Vol. 182, Issue 2, pp. 418477. November, 2002.

[3] A. Bertram. "Elasticity and Plasticity of Large Deformations". Springer Verlag. Berlin, Germany. 2005.

[4] P. Castaneda, J. Telega and B. Gambin. "Nonlinear Homogenization and its Application to Composites, Polycrystals and Smart Materials". Proceedings of the NATO Advanced Research Workshop, NATO Science Series II: Mathematics, Physics and Chemistry 170. Springer. Netherland. 2004.

[5] W. Chan and A. George. "A linear time implementation of the Reverse Cuthill-McKee algorith". BIT Numerical Mathematics. Vol. 20, pp. 8-14. 1980.
[6] E. Cuthill and J. McKee. "Reducing the bandwidth of sparse symmetric matrices". Proceedings of the $196924^{\text {th }}$ National Conference of the ACM, ACM Press, pp. 157-172. New York, N.Y., USA. 1969.

[7] S.Y. Fialko. "The nested substructure method for solving large finite-element systems as applied to thin-walled shells with high ribs". International Applied Mechanics. Vol. 39, Issue 3, pp. 324-331. March, 2003.

[8] J. Fish and R. Fan. "Mathematical homogenization of nonperiodic heterogeneous media subjected to large deformation transient loading". International Journal for Numerical Methods in Engineering. Vol. 76, pp. 1044-1064. 2008.

[9] F. Fritzen and T. Böhlke. "Influence of the type of boundary conditions on the numerical properties of unit cell problems". Technische Mechanik. Vol. 30, Issue 4, pp.354-363. 2010.

[10] N. Gibbs, W. Poole and P. Stockmeyer. "An algorithm for reducing the bandwidth and profile of a sparse matrix". Society for Industrial and Applied Mathematics Journal on Numerical Analysis. Vol. 13, Issue 2, pp. 236-250. April, 1976.

[11] T. Kanit, S. Forest, I. Galliet, V. Mounoury and D. Jeulin. "Determination of the size of the representative volume element for random composites: statistical and numerical approach". International Journal of Solids and Structures. Vol. 40, Issue 13, pp. 3647-3679. 2003.

[12] I.-S. Liu. "Continuum Mechanics". Springer Verlag. Berlin, Germany. 2002.

[13] S. Mesarovic and J. Padbidri. "Minimal kinematic boundary conditions for simulations of disordered microstructures". Philosophical Magazine. Vol. 85, Issue 1, pp. 65-78. January, 2005.

[14] S. M. Sloan. "An algorithm for profile and wavefront reduction of sparse matrices". International Journal for Numerical Methods in Engineering. Vol. 23, Issue 2, pp. 239-251. February, 1985.

[15] T.I. Zohdi and P. Wriggers. "Computational Micro-macro Material Testing". Archives of Computational Methods in Engineering. Vol. 8, Issue 2, pp. 131-228. 2001. 